\title{
A Rare Case of Peritoneal Tuberculosis
}

\author{
Wan-Ling Alyssa Chiew ${ }^{\mathrm{a}, \mathrm{b}}$, Jia Xuan Jolene Liu ${ }^{\mathrm{a}}$, Li Tserng Teo ${ }^{\mathrm{a}}$
}

\begin{abstract}
Peritoneal tuberculosis (TB) is a rare extra-pulmonary presentation of TB, caused by strains of mycobacteria, usually Mycobacterium tuberculosis. The clinical presentation of peritoneal TB can be extremely varied, making it a huge diagnostic challenge. Herein, we report a case of a 62-year-old Chinese female with no previous exposure to $\mathrm{TB}$, presenting with 1-day history of per-rectal bleeding and abdominal pain. The diagnosis was eventually made with a combination of blood investigations, computerized tomography scans and histological analysis of peritoneal nodules found on diagnostic laparoscopy, which confirmed necrotizing granulating inflammation. She was subsequently started on anti-TB medications promptly without any further complications and recovered uneventfully.
\end{abstract}

Keywords: Peritoneal tuberculosis; Raised CA-125; Surgery

\section{Introduction}

Mycobacterium tuberculosis is endemic in Singapore, with an incidence rate of 36.9 per 100,000 population in 2014. Majority $(84.5 \%)$ of cases had pulmonary tuberculosis (TB) with or without extra-pulmonary involvement, while the remainder $(15.5 \%)$ had exclusively extra-pulmonary TB. The most common site of extra-pulmonary TB was the lymphatic system followed by the pleura [1].

Most often, extra-pulmonary TB results from reactivation of latent disease established by hematogenous spread during primary pulmonary infection. Clinical manifestations of abdominal TB are rather non-specific, often mimicking other abdominal diseases. Up to $88 \%$ of patients with abdominal TB present with abdominal pain, with associated symptoms of weight loss (75\%), anorexia (62\%), and night sweats (54\%) [2] (Table 1).

Risk factors for peritoneal TB include immunocompromised states such as human immunodeficiency virus (HIV) infection, poorly controlled diabetes mellitus, alcoholic liver

Manuscript accepted for publication March 04, 2016

aDepartment of General Surgery, Tan Tock Seng Hospital, Singapore ${ }^{b}$ Corresponding Author: Wan-Ling Alyssa Chiew, Department of General Surgery, Tan Tock Seng Hospital, 11 Jalan Tan Tock Seng, 308433, Singapore. Email: alyssachiew@gmail.com

doi: http://dx.doi.org/10.14740/jmc2449w disease, underlying malignancies, following treatment with anti-tumor necrosis factor (TNF), and patients undergoing peritoneal dialysis.

\section{Case Report}

A 62-year-old Chinese female was admitted under the Department of General Surgery for 1-day history of per-rectal bleeding and mechanical fall. Past medical history included a left middle cerebral artery stroke associated with right hemiparesis a few years ago, hypertension and hyperlipidemia. She had no significant weight loss, night sweats, fever and productive cough, and also had no known exposure to TB, family history or risk factors for TB.

During physical examination, patient was alert, afebrile, normotensive and non-tachycardia. Abdomen was soft, nontender with no palpable masses and no signs of peritonism. Digital rectum examination showed altered blood, but no melena or masses were felt. Full blood count revealed normocytic, normochromic anemia with hemoglobin of $7.6 \mathrm{~g} / \mathrm{dL}$. Coagulation profile, liver function tests and electrolytes were all normal. Plain chest and abdominal radiography showed no obvious abnormalities.

In view of the clinical picture, the initial impression was to exclude rectal bleeding either secondary to diverticular bleeding or gastrointestinal malignancy. With suspicion of malignancy, serum tumor markers were sent off. CA-125 level was elevated at $99 \mathrm{U} / \mathrm{mL}(<35.1 \mathrm{U} / \mathrm{mL})$ while other tumor markers were normal, with carcinoembryonic antigen (CEA) of $5 \mu \mathrm{g} / \mathrm{L}$ $(<5 \mu \mathrm{g} / \mathrm{L})$ and alphafetoprotein (AFP) of $3 \mu \mathrm{g} / \mathrm{L}(<9 \mu \mathrm{g} / \mathrm{L})$. Given that CA-125, ovarian epithelial carcinoma with possible metastasis was suspected. A computerized tomography (CT) of the abdomen and pelvis was done (Fig. 1), which revealed an irregular pelvic mass that appeared to arise from the sig-

Table 1. Clinical Features of Abdominal Tuberculosis [2]

Abdominal pain

Abdominal distension

Weight loss

Ascites

Diarrhea

Nausea and vomiting

Abdominal mass

"Doughy" abdomen (rare) 


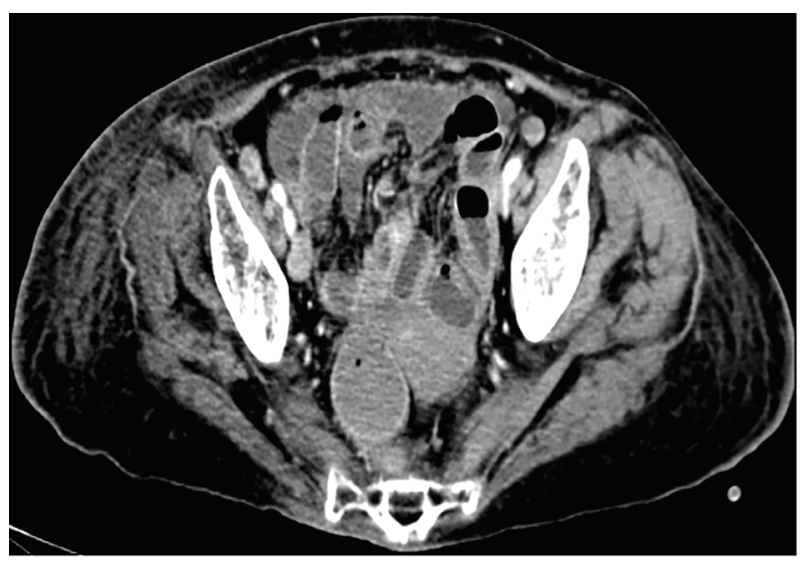

Figure 1. CT scan revealing irregular pelvic mass.

moid colon or uterus; mild ascites; multiple enlarged mesenteric, para-aortic, aortocaval and bilateral external iliac lymph nodes; multiple sub-centimeter pulmonary nodules with bilateral small pleural effusions. Then, a flexible sigmoidoscopy (Fig. 2) was performed to obtain histology. Findings revealed a suspected extrinsic compression at $30 \mathrm{~cm}$ and no colonic lesions noted.

In view of possible advanced ovarian carcinoma, the patient was transferred under the care of the Department of Obstetrician and Gynaecology. An ultrasound (US) of the pelvis performed showed only subserosal fibroid $4.7 \mathrm{~cm}$, and endometrial thickness not thickened. A magnetic resonance imaging (MRI) of abdomen and pelvis performed showed irregular lesions in proximity to sigmoid colon, associated with mild wall thickening of sigmoid colon and adjacent small bowel loops. Some multiple enlarged mesenteric, para-aortic and bilateral external iliac lymph nodes showed necrosis. Mild ascites with mesenteric stranding and nodularity of left paracolic gutter were seen. After a discussion between the two specialties and multiple investigations, impression was possibly peri-

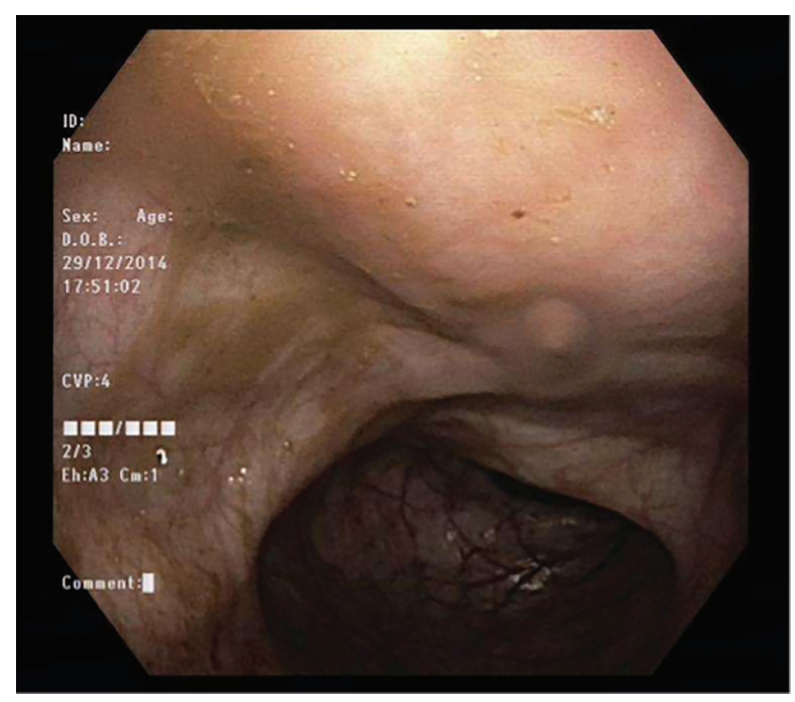

Figure 2. Flexible sigmoidoscopy suggestive of a possible extrinsic compression by a pelvic mass.

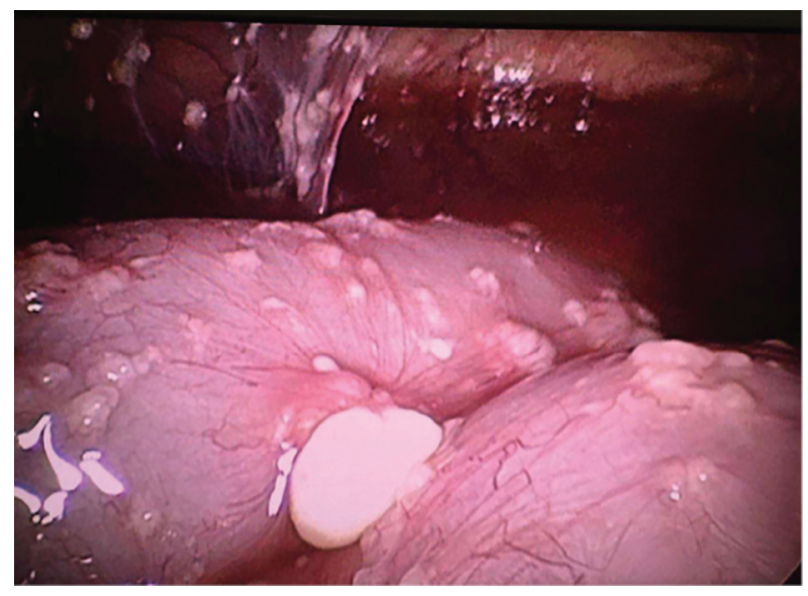

Figure 3. Diagnostic laparoscopy revealed miliary seedlings on peritoneum and serosal surface of bowel with dense adhesions.

toneal metastases from the gastrointestinal tract (GIT) rather than gynecological-related, or possible peritoneal granulomatous inflammation such as mycobacterium tuberculosis.

A diagnostic laparoscopy was performed subsequently. Multiple military seedlings were noted in the peritoneum and on serosal surface of bowel. Ascites with dense adhesions were also seen (Fig. 3). There were no malignant cells seen in the peritoneal fluid. Cultures of peritoneal fluid and nodules eventually grew Mycobacterium tuberculosis complex. Histology of the peritoneal nodules returned as necrotizing granulomatous inflammation. The two previous sets of sputum acid-fast bacilli (AFB) smear were negative; however, cultures were positive for TB. Biopsy of a $3 \mathrm{~mm}$ sigmoid polyp, found 20 $\mathrm{cm}$ from the anal verge, revealed low grade dysplasia. Patient was then referred to respiratory medicine physician and started on anti-tuberculous medications, rifampicin, isoniazid, ethambutol, pyrazinamide and pyridoxine promptly. The patient recovered subsequently.

\section{Discussion}

Peritoneal TB is a rare extra-pulmonary presentation of TB presenting with non-specific signs and symptoms. The diagnosis of peritoneal TB can be especially difficult in elderly female patients, as seen in our case, where they usually present with vague abdominal symptoms and raised CA-125 markers, in which case the first differential working diagnosis is usually to rule out an underlying gynecological malignancy.

The diagnosis of peritoneal TB can be established by one of the following criteria: 1) definitive diagnosis: histologic and microbiologic evidence of Mycobacterium tuberculosis, the presence of granulomas with caseous necrosis, successful culture of $M$. tuberculosis from the tissue specimen, or the presence of documented TB in another site with typical operative findings and granulomas; or 2) clinical diagnosis: clinical and radiological features of abdominal TB (Table 2) [3], responding to anti-tuberculous medication in the absence of definitive diagnosis.

Although the tumor marker CA-125 has poor sensitivity 
Table 2. Common Findings for Peritoneal Tuberculosis Using the Various Diagnostic Modalities [3]

\begin{tabular}{lll}
\hline Ultrasound findings & CT findings & Laparoscopic findings \\
\hline $\begin{array}{l}\text { Abdominal lymphadenopathy } \\
\text { with hypoechogenic centers }\end{array}$ & $\begin{array}{l}\text { Abdominal lymphadenopathy with low } \\
\text { attenuation centers and an enhanced rim } \\
\text { Localized ascites }\end{array}$ & $\begin{array}{l}\text { Thickened, hyperemic, occasionally } \\
\text { hemorrhagic, inflamed peritoneum }\end{array}$ \\
$\begin{array}{l}\text { Septations } \\
\text { Fine strands }\end{array}$ & Thickened mesentery and bowel wall & $\begin{array}{l}\text { Diffuse, white-yellowish uniform (3 - 5 mm) tubercular } \\
\text { deposits on peritoneum, omentum and organs }\end{array}$ \\
Mass lesions & & \\
\hline
\end{tabular}

and specificity, it has been shown to be raised in cases of TB peritonitis, mimicking ovarian epithelial carcinoma as CA-125 is produced by normal epithelial cells (peritoneum, pleural and pericardium) when inflamed. Only $20 \%$ of women with abnormal CA-125 results were eventually diagnosed with ovarian malignancy in a retrospective study conducted by Moss and his colleagues [4]. CA-125 has also been shown to be useful in monitoring treatment response as seen by the decline in CA125 paralleling clinical response following anti-TB treatment [5].

Diagnosis of TB peritonitis is often delayed or missed due to its non-specific clinical presentation, long incubation period for the AFB cultures which usually takes up to $4-8$ weeks, and high false negative rates for AFB smears. This can not only result in a delay in starting treatment but also result in inappropriate medical decisions such as unnecessary resection of reproductive organs if mistaken for possible ovarian carcinoma with peritoneal metastasis or exploratory laparotomy if diagnosis is still unclear for a potentially medically treated condition. An effective therapy and increased survival rates have been associated with early diagnosis of peritoneal TB [6].

\section{Conclusions}

Diagnosis of peritoneal TB depends on a constellation of clinical, radiological and histopathological findings. The gold standard for diagnosis is still diagnostic laparoscopy with classical findings of peritoneal studdings of tubercles (military seeds) with dense adhesions, and histological findings of caseating granulomas with epithelioid and Langhan's type giant cells. Hence, it is important for physicians to keep a high index of suspicion for peritoneal TB in patients who present with vague abdominal symptoms that are not in keeping with classical clinical or radiological findings, and be able to recognize the common radiological and laparoscopic appearances to ensure early and accurate diagnosis of this increasingly common disease.

\section{Learning points}

1) A high clinical suspicion is required for an early and accurate diagnosis of TB peritonitis.
2) Raised CA-125 markers can be found in cases of TB peritonitis, thus mimicking ovarian epithelial carcinoma.

3) Abdominal TB should be considered as a differential diagnosis for patients who are from TB endemic countries or travelled recently to such countries presenting with non-specific abdominal complaints.

4) Surgeons should be able to recognize the common radiological and laparoscopic findings of peritoneal TB and include as a differential diagnosis for abdominal symptoms.

5) Diagnosis laparoscopy is the ideal modality for early diagnosis.

\section{Conflict of Interest}

The authors have no conflict of interest to declare.

\section{Grant Support}

None.

\section{References}

1. Ministry of Health $(\mathrm{MOH})$ Singapore, Communicable Diseases Surveillance in Singapore Annual Report. 2014.

2. Underwood MJ, Thompson MM, Sayers RD, Hall AW. Presentation of abdominal tuberculosis to general surgeons. Br J Surg. 1992;79(10):1077-1079.

3. Rasheed S, Zinicola R, Watson D, Bajwa A, McDonald PJ. Intra-abdominal and gastrointestinal tuberculosis. Colorectal Dis. 2007;9(9):773-783.

4. Moss EL, Hollingworth J, Reynolds TM. The role of CA125 in clinical practice. J Clin Pathol. 2005;58(3):308312.

5. Mas MR, Comert B, Saglamkaya U, Yamanel L, Kuzhan O, Ateskan U, Kocabalkan F. CA-125; a new marker for diagnosis and follow-up of patients with tuberculous peritonitis. Dig Liver Dis. 2000;32(7):595-7

6. Lataifeh I, Matalka I, Hayajneh W, Obeidat B, Al Zou'bi H, Abdeen G. Disseminated peritoneal tuberculosis mimicking advanced ovarian cancer. J Obstet Gynaecol. 2014;34(3):268-71 